\title{
UMA REVISÃO DOS ESTUDOS SOBRE A FALA DIRIGIDA À CRIANÇA E SUAS IMPLICAÇÕES PARA A AQUISIÇÃO INICIAL DO VOCABULÁRIO
}

\author{
Poliana Gonçalves Barbosa* \\ Universidade de Alberta \\ Edmonton, Alberta, Canadá
}

\author{
Cláudia Cardoso-Martins \\ Universidade Federal de Minas Gerais \\ Belo Horizonte, Minas Gerais, Brasil
}

\begin{abstract}
Resumo: Pesquisadores motivados pelo argumento de Chomsky sobre a pobreza do input linguístico têm investigado as características da fala dirigida à criança (FDC) e seu impacto no desenvolvimento da linguagem infantil. Nesta retrospectiva, revemos estudos que investigaram a relação entre a FDC e o desenvolvimento inicial do vocabulário pela criança. Os resultados sugerem que variações no estilo, no comprimento médio dos enunciados e na frequência e variedade de palavras na FDC têm um impacto na aquisição inicial do vocabulário infantil. Contudo, esses resultados devem ser interpretados com cuidado. De modo geral, apenas estudos correlacionais investigaram a contribuição da FDC para o desenvolvimento do vocabulário. Além disso, a maioria deles foi conduzida com crianças falantes do inglês. Estudos experimentais e incluindo crianças falantes de outras línguas são claramente necessários para investigar essa questão.
\end{abstract}

Palavras-chave: Aquisição da linguagem. Fala dirigida à criança. Vocabulário.

1 INTRODUÇÃO

Ao longo dos últimos 40 anos, vários pesquisadores têm investigado o papel desempenhado pela fala dirigida à criança (FDC) no desenvolvimento da linguagem inicial. Esses estudos foram, em grande parte, motivados pelo argumento da "pobreza do estímulo" (CHOMSKY, 1965), segundo o qual o input linguístico não é suficientemente informativo para a aquisição da linguagem.

Para Chomsky, a informação contida no input linguístico é escassa e, muitas vezes, incompleta e incorreta, não sendo suficiente para permitir que uma criança alcance a competência linguística de um adulto com base apenas em mecanismos de aprendizagem gerais como, por exemplo, a imitação e a associação entre estímulos e respostas. Ao contrário, Chomsky (1995) propõe que as crianças, ao nascerem, possuem um órgão/módulo linguístico composto por princípios (conhecimentos linguísticos

\footnotetext{
Mestre em Psicologia. Doutoranda na Universidade de Alberta - Canadá. Email: polianabarbosa87@gmail.com ou poliana@ualberta.ca

** Doutora em Psicologia. Email: cardosomartins.c@gmail.com
} 
abstratos, compartilhados por todas as línguas humanas, e.g., o princípio de que todas as sentenças têm um sujeito) e parâmetros (conhecimentos que variam entre as línguas, e.g., o fato de o sujeito gramatical poder ou não ser implícito nas sentenças). Para o autor nativista, essa estrutura inata seria a única solução lógica para o problema imposto pela natureza do input linguístico (CHOMSKY, 1997). Sob essa ótica, o input linguístico da criança seria apenas um gatilho. Ou seja, ele indicaria para a criança quais regras aplicam-se à sua língua natal (CHOMSKY, 1997). Em outras palavras, para Chomsky, a aquisição da linguagem é algo que acontece às crianças e não algo que elas fazem.

Entretanto, muitos pesquisadores têm questionado esse ponto de vista nativista da aquisição da linguagem. Particularmente, um número expressivo de autores tem colocado em xeque a existência de representações inatas e abstratas de categorias gramaticais (e.g., TOMASELLO, 2011). Na opinião desses pesquisadores, a estrutura linguística emerge do uso da linguagem:

muitas das produções de mais de uma palavra das crianças não são estruturadas por regras abstratas, mas sim por esquemas linguísticos de uma natureza muito mais concreta - os quais têm sido chamados de esquemas baseados no item (ou ilhas de construção) ligados a um conteúdo lexical concreto. Esses esquemas se tornam abstratos apenas gradualmente [...]. (TOMASELLO, 2006, p. 2) ${ }^{1}$.

Naturalmente, essa abordagem da aquisição e desenvolvimento da linguagem atribui um papel muito mais relevante ao input linguístico do que Chomsky e outros expoentes nativistas vêm atribuindo. De fato, estudos que investigaram o ambiente linguístico de crianças pequenas sugerem que, ao contrário da descrição de Chomsky, a FDC possui várias características que podem desempenhar um papel-chave na aquisição da linguagem. Por exemplo, ela é constituída por falas curtas, repetitivas e gramaticalmente corretas (FERGUSON, 1977; FERNALD, 1989; NEWPORT; GLIETMAN; GLEITMAN, 1977; SNOW, 1972). Ela também funciona como um dispositivo para atrair a atenção de bebês (ALBIN; ECHOLS, 1996; FERNALD; SIMON, 1984), os quais preferem a FDC à fala comumente dirigida ao adulto (FDA) desde os primeiros meses de vida (COOPER; ASLIN, 1990; FERNALD, 1985). Esse fato, especificamente, auxiliaria as crianças a prestarem atenção ao código linguístico (BREDVAD-JENSEN, 1996; FERNALD; MAZZIE, 1991). Outra característica importante da FDC é o fato de que ela se ajusta/adapta às habilidades linguísticas das crianças. Por exemplo, quanto maior o MLU (comprimento médio do enunciado = total de palavras/morfemas dividido pelo total de sentenças) das sentenças produzidas pela criança, maior é o MLU da fala dirigida a ela por suas mães (NEWPORT et al., 1977; ROY; FRANK; ROY, 2009). Juntas, essas características sustentam o argumento de alguns autores de que a FDC poderia ser concebida como um dispositivo para o ensino e aquisição da linguagem (FERNALD; MAZZIE, 1991; FERGUSON, 1977), questionando assim a proposição da pobreza do estímulo.

\footnotetext{
${ }^{1}$ Tradução livre do autor: "[...] many of young children's early multi-word productions are not structured by abstract rules but rather by linguistic schemas of a much more concrete nature - what have been called item-based schemas (or constructional islands) tied to concrete lexical content. These schemas become abstract only gradually [...]."
} 
Essa fala específica dirigida à criança foi inicialmente chamada de manhês (motherese) por Newport (1975). Há, no entanto, evidência de que esse registro especial não é algo exclusivo das mães, mas é usado por praticamente qualquer adulto, e até mesmo por crianças mais velhas, para se dirigir aos bebês e crianças pequenas (FERGUSON, 1977). Tal constatação fez com que esse estilo comunicativo fosse renomeado para fala dirigida ao infante (FDI $\sim$ até 1 ano de idade) ou fala dirigida à criança (FDC $\sim$ a partir de 1 ano). Neste trabalho adotaremos a nomenclatura FDC quando estivermos nos referindo ao manhês, embora alguns dos estudos que serão apresentados aqui tenham investigado crianças com menos de 1 ano de idade.

Presente em quase todas as culturas estudadas, a FDC não possui exatamente as mesmas características nos vários idiomas existentes. Apesar disso, sua função comunicativa de expressão e socialização (FERGUSON, 1977), sua função afetivaemocional (BROOKS; KEMPE, 2012) e seu contorno melódico, (MAHDHOUI; CHETOUANI; ZONG, 2008) são aparentemente comuns a todos os idiomas.

Ao longo do tempo, algumas revisões de literatura sobre a FDC foram realizadas (e.g., SNOW, 1977; HOFF-GINSBERG; SHATZ, 1982; GLEITMAN; NEWPORT; GLEITMAN, 1984; SODERSTROM, 2007). De um modo geral, essas revisões investigaram o impacto da FDC sobre o desenvolvimento gramatical. No presente trabalho, descrevemos os resultados de estudos que avaliaram o impacto da FDC na aquisição do vocabulário.

Poucos estudos investigaram se as características da FDC poderiam contribuir para a aquisição do vocabulário inicial pelas crianças. No Brasil, não encontramos nenhum estudo que tenha abordado diretamente essa questão. A imensa maioria dos estudos aqui realizados investigou os aspectos comunicativo-funcional, afetivoemocional e social presentes na fala materna dirigida à criança (AQUINO; SALOMÃO, 2005; BARROS; CAVALCANTE, 2011; BRAZ; SALOMÃO, 2002; DADALTO; GOLDFELD, 2006; FERREIRA, 2001; FONSÊCA; SALOMÃO, 2005; PÊSSOA; SEIDL-DE-MOURA, 2008; PÊSSOA; SEIDL-DE-MOURA, 2011; PÊSSOA; SEIDLDE-MOURA; OLIVA, 2008; VERÁS; SALOMÃO, 2005), e nenhum investigou o impacto desses aspectos no desenvolvimento da linguagem pela criança.

Para aprender uma palavra nova, as crianças devem ser capazes de identificá-la no fluxo contínuo da fala e conectá-la a objetos ou eventos da situação presente (HOFF, 2005; TOMASELLO, 2011; WAXMAN; LEDDON, 2011). Em outras palavras, as crianças precisam extrair a palavra da fala, identificar a entidade relevante no mundo à qual o falante se refere, e estabelecer um mapeamento entre elas.

Em geral, os estudos relacionados à FDC e suas influências no vocabulário inicial podem ser agrupados em duas categorias diferentes: aqueles que estudaram (1) o problema da segmentação da fala em palavras, e aqueles que avaliaram (2) os efeitos da FDC na aquisição do vocabulário pelas crianças. Esses estudos são discutidos a seguir. 
Como observamos anteriormente, para aprender uma língua, os bebês precisam identificar as palavras. Isso constitui um problema, uma vez que as palavras não são normalmente separadas umas das outras no input linguístico (BROOKS; KEMPE, 2012). Pelo contrário, o input linguístico é um contínuo, e os limites onde uma palavra começa e a outra termina são obscuros.

Há evidência de que as crianças utilizam algumas pistas para identificar as palavras no input linguístico, incluindo, por exemplo, os contornos prosódicos típicos de palavras isoladas (entonação característica que as ressaltam quando inseridas em sentenças), e as fronteiras de unidades prosódicas (e.g., entonação descendente, alongamento das sílabas e pausas) (GOUT; CHRISTOPHE, 2006). Aparentemente, as crianças também são sensíveis a variações alofônicas, por exemplo, elas percebem quando um mesmo fonema é pronunciado de modo diferente dependendo da posição que ocupa na palavra e nas sílabas - "Por exemplo, tanto o /t/ como o /r/ de 'night rates' são diferentes dos de 'nitrates' (o /t/ é aspirado, distenso e retroflexo em 'nitrates', e não aspirado e tenso em 'night rates'; o /r/ é surdo em 'nitrates', mas é sonoro em 'night rates')" (GOUT; CHRISTOPHE, 2006, p. 109). Elas também parecem perceber a existência de algumas regularidades distribucionais no discurso, isto é, que algumas sequências de fonemas - /fh/ - só ocorrerem na fronteira entre palavras - belief /bı'lif/ e house /havs/; enquanto outras - /ft/ - só ocorrerem em uma mesma palavra - heft /heft/ (JUSCZYK, 1999). Por exemplo, Saffran, Aslin e Newport (1996) mostraram que bebês de 8 meses são capazes de extrair informações sobre os limites das palavras apenas com base na sequência estatística de palavras em uma fala concatenada, e na probabilidade de elas coocorrerem dentro de uma mesma sentença.

De uma maneira geral, os estudos que investigaram a capacidade de segmentação da fala pelas crianças empregaram o Paradigma da Escuta Preferencial - Headturn Preference Procedure (ver KEMLER-NELSON et al. 1995, para mais detalhes), o qual pode ser utilizado com bebês de 4 a 18 meses (BRUM-DE-PAULA, 2010; NAME; CORRÊA, 2006). Nesse paradigma o bebê é inicialmente familiarizado a um estímulo sonoro apresentado por caixas de sons à sua frente. Posteriormente, lhe é apresentado um estímulo novo, alternadamente com o estímulo familiarizado, por caixas de sons laterais. Partindo do pressuposto de que os bebês demonstram preferência pela novidade, quando eles olham mais tempo na direção do estímulo novo, deduz-se que eles reconheceram (processaram) o estímulo apresentado anteriormente (dito familiarizado) e o diferenciaram do estímulo novo.

Empregando esse paradigma, algumas pesquisas indicam que, a partir de 7 meses e meio de idade, as crianças parecem ser capazes de segmentar a fala no nível de palavras com base apenas em informação de natureza puramente estatística (regularidades distribucionais), i.e., são capazes de fazê-lo mesmo quando os aspectos prosódicos e as pausas presentes na fala são removidos computacionalmente (ASLIN; SAFFRAN; NEWPORT, 1998; FERNALD; MCROBERTS; SWINGLEY, 2001; JUSCZYK, 1999; JUSCZYK; ASLIN, 1995; KANPEM et al., 2008; SAFFRAN et al., 1996). 
Embora as crianças sejam capazes de segmentar o fluxo da fala no nível de palavras baseando-se apenas em regularidades estatísticas presentes no input linguístico, é possível que algumas características da FDC como, por exemplo, sua prosódia exagerada e o fato de a fala dirigida à criança pequena referir-se normalmente ao aqui-eagora (SNOW, 1971), possam facilitar esse processo. De acordo com Fernald e Mazzie (1991), essas características provavelmente facilitam a identificação, pela criança, da palavra e de seu referente.

A maior efetividade da FDC, quando comparada à FDA, para auxiliar na segmentação do fluxo da fala em palavras, foi mostrada por Thiessen, Hill e Saffran (2005). Os autores criaram 12 enunciados que começavam e terminavam da mesma forma e eram compostos pelas mesmas pseudopalavras, porém apresentadas em ordens diferentes (e.g., "mo dibo lagoti nifopa kuda fa", "mo nifopa lagoti kuda dibo fa"). Esses enunciados foram gravados de acordo com os estilos típicos da FDC e da FDA por uma mesma pessoa. Através de um programa de computador, os dois tipos de falas (FDA e FDC) foram equiparados em seu comprimento e amplitude, sendo retiradas as pausas entre as pseudopalavras e suas sílabas, mantendo-se apenas a diferença prosódica entre eles. $\mathrm{O}$ experimento foi feito com 40 crianças falantes do inglês entre 6,5 e 7,5 meses, das quais metade escutou os enunciados em FDC e metade em FDA, por um minuto. Posteriormente, na fase teste, elas escutaram, alternadamente, listas compostas dessas pseudopalavras pronunciadas separadamente (e.g., "lagoti", "nifopa") e listas compostas de pseudopalavras diferentes, mas formadas pelas sílabas presentes nas pseudopalavras familiares (e.g., "tinifo", "pakuda", etc.). As crianças que haviam escutado a FDA não mostraram preferência por nenhum tipo de estímulo. Por outro lado, as crianças que ouviram a FDC mostraram preferência pelas pseudopalavras familiares. Essa preferência sugere que, ao contrário dos bebês na condição FDA, os bebês da condição FDC diferenciaram os estímulos familiares dos demais, indicando que eles foram capazes de identificá-los no contínuo da fala. No entanto, é possível que os bebês ainda não estivessem totalmente familiarizados aos estímulos familiares, daí sua preferência por eles durante a fase de teste. Frente a esses resultados, os autores realizaram um novo experimento. Nesse segundo estudo, 50 crianças entre 7,5 e 8,5 meses foram expostas aos mesmos estímulos do estudo anterior, contudo pelo dobro de tempo (2 minutos). Desta vez, as crianças que ouviram a FDC mostraram uma clara preferência pelas pseudopalavras não familiares. Novamente, o grupo de crianças expostas à FDA não mostrou preferência por qualquer tipo de estímulo. Os autores concluíram que a FDC facilita a segmentação da fala por meio da informação prosódica. De acordo com Thiessen et al. é possível que as crianças expostas à FDC tenham prestado mais atenção aos estímulos e, como resultado, tenham sido mais capazes de armazená-los na memória do que as demais crianças. Conforme observamos anteriormente, há evidência de que as crianças preferem a FDC à FDA (COOPER; ASLIN, 1990; FERNALD, 1985).

É possível que outras características da FDC também contribuam para a segmentação da fala em palavras pelas crianças. Cameron-Faulkner, Lieven e Tomasello (2003) investigaram a fala materna dirigida a doze crianças entre 1,9 e 2,6 anos, em um contexto de brincadeira livre. Todos os enunciados maternos dirigidos à 
criança foram transcritos para posterior análise, com exceção daqueles referentes a rotinas sociais ("bom dia", "oi", "tchau"), totalizando 16.903 enunciados. Esses foram codificados como fragmentos (palavras isoladas, sintagmas nominais, sintagmas verbais, sintagmas preposicionais e outras multipalavras) e sentenças completas (interrogativas, imperativas, declarativas, cópulas, e frases complexas). CameronFaulkner et al. (2003) constataram que 22\% dos enunciados da FDC correspondiam apenas a fragmentos. Esses resultados de Cameron-Faulkner et al. (2003) reforçaram os resultados do estudo de Newport et al. (1977), os quais também evidenciaram uma proporção considerável de enunciados fragmentados (17\%) na FDC. De acordo com Cameron-Faulkner et al., é possível que essa alta incidência de fragmentos na FDC $(17 \%$ a $22 \%$ ) auxilie as crianças a identificar palavras familiares no contínuo da fala, facilitando dessa forma o problema da segmentação da fala.

Essa questão foi examinada diretamente por Bortfeld et al. (2005). Utilizando o Paradigma da Escuta Preferencial (descrito anteriormente), os autores mostraram que bebês de 6 meses de idade são capazes de usar palavras frequentes na FDC (e.g., o nome da própria criança) para identificar novas palavras que aparecem antes ou depois da palavra familiar no input linguístico. Os autores mostraram que as crianças reconheciam palavras novas quando essas seguiam seus nomes próprios no enunciado, mas não quando elas seguiam um outro nome qualquer (e.g., Maggie's/Lola's bike had big, black wheels - A bicicleta da Maggie/Lola tinha rodas pretas grandes). O mesmo resultado foi encontrado quando as palavras novas eram precedidas pela palavra mommy (mamãe), que é também muito frequente no input linguístico dos bebês (e.g., The girl laughed at Mommy's feet - A garota riu do pé da mamãe).

Uma vez que a FDC auxilia o bebê a segmentar a fala, não seria surpreendente se ela também contribuísse para aquisição inicial do vocabulário.

\section{IMPACTO DA FALA DIRIGIDA À CRIANÇA NA AQUISIÇÃO INICIAL DO VOCABULÁRIO}

A seguir, descrevemos os resultados de estudos que investigaram o papel desempenhado pela FDC na aquisição inicial do vocabulário infantil. A maioria desses estudos foi de natureza correlacional, ou seja, avaliou a relação entre variações na FDC e variações no vocabulário da criança. Conforme descrevemos a seguir, aparentemente apenas o estudo de Singh et al. (2009) utilizou uma metodologia experimental para investigar essa questão.

\subsection{UM ESTUDO EXPERIMENTAL}

Singh et al. (2009) usaram o Paradigma da Escuta Preferencial para investigar o impacto da FDC, mais especificamente, dos seus aspectos prosódicos, no armazenamento e recuperação de palavras da memória. Durante a fase de familiarização, trinta e dois bebês de 7,5 meses de idade ouviram dois pares de palavras (bike-hat e tree-pear), um deles enunciado no estilo FDC (e.g., "His bike had big black wheels"; "The hat was blue and white") e o outro no estilo FDA (e.g., "The tree was a 
hundred years old"; "She ate the whole pear"). Cada palavra aparecia 15 vezes em frases diferentes. Vinte e quatro horas depois, metade dos bebês ouviu passagens em FDC (um quarto das quais continha as palavras familiarizadas em FDC, um quarto as palavras familiarizadas em FDA e a metade restante contendo palavras novas) e a outra metade ouviu as mesmas passagens, mas no estilo FDA. Os resultados mostraram que os bebês preferiram os enunciados contendo as palavras familiarizadas em FDC (independentemente de como essas palavras eram apresentadas durante a fase teste, no estilo FDC ou FDA) do que aqueles enunciados contendo as palavras familiarizadas em FDA ou as palavras não familiarizadas. Ou seja, eles foram capazes de discriminar o estímulo familiar do estímulo novo. Singh et al. (2009) concluíram que a FDC auxilia a criança não apenas na recuperação da palavra aprendida após um longo período, mas também parece proporcionar representações mnemônicas mais genéricas, uma vez que as crianças reconheceram a palavra familiarizada em FDC, mesmo quando ela foi posteriormente apresentada em FDA.

\subsection{OS ESTUDOS CORRELACIONAIS}

Dentre os estudos que utilizaram métodos correlacionais, o trabalho de Nelson (1973) tem sido frequentemente citado na literatura. A autora avaliou a linguagem de dezoito mães, às suas crianças, quando estas estavam com 13 e 14 meses de idade. Dois estilos de comunicação foram identificados na interação das mães e suas crianças. No estilo diretivo, as mães faziam referências frequentes ao comportamento da criança, procurando dirigir sua atenção e comportamento, e utilizavam poucas perguntas; o estilo referencial, por outro lado, caracterizava-se por nomeações frequentes de objetos, além de muitas perguntas e poucos comandos/diretivos. Diversas medidas da FDC (e.g. conteúdo, forma e feedback) foram correlacionadas com variações na aquisição de vocabulário pelas crianças ao longo do segundo e do terceiro ano de vida. De acordo com Nelson, o estilo diretivo e as referências ao comportamento da criança aos 13 meses se correlacionaram negativamente com as medidas de vocabulário das crianças durante o segundo ano de vida. Contrariamente, o estilo referencial se correlacionou positivamente com o tamanho do vocabulário infantil aos 24 meses.

Newport et al. (1977) avaliaram diversos aspectos da FDC e sugeriram que a FDC apresenta um impacto modesto no crescimento da linguagem, incluindo o vocabulário. Quinze mães e suas filhas participaram do estudo. A fala materna dirigida à criança e a própria fala da criança foram obtidas em duas ocasiões, com duração de duas horas cada, separadas por um intervalo de seis meses. Na primeira ocasião, as crianças tinham entre 12 e 27 meses de idade. Nas duas ocasiões, os enunciados maternos foram classificados em função das seguintes categorias: correção gramatical, comprimento das sentenças em morfemas, tipos de sentença, inteligibilidade, repetição, expansão ou imitação da fala da criança, e dêixis. O mesmo foi feito em relação aos enunciados da criança, que foram classificados quanto ao comprimento em morfemas, comprimento e frequência de sintagmas nominais, comprimento e frequência de sintagmas verbais, flexões de sintagmas nominais, estruturas auxiliares e tamanho do vocabulário expressivo. As medidas da fala materna foram correlacionadas às medidas da fala da 
criança, separadamente para cada ocasião. Além disso, os autores correlacionaram a fala materna na primeira ocasião com a fala infantil na segunda. Poucas correlações foram encontradas entre a FDC e o vocabulário da criança, i.e., apenas a frequência em que as mães diziam "yes", "mm-hum" ou "no" em resposta à fala das crianças se correlacionou positivamente com o vocabulário infantil.

Como descrito, Newport et al. (1997) avaliaram a correlação entre tipos de enunciados linguísticos diferentes e o vocabulário da criança, no entanto, uma medida mais apropriada poderia ser o próprio vocabulário da mãe medido em termos de frequência e diversidade de palavras. Huttenlocher et al. (1991) mostraram que em uma amostra de vinte e duas crianças falantes do inglês, variações na quantidade e diversidade de palavras presentes na linguagem materna correlacionam-se positivamente com o crescimento do vocabulário infantil entre os 14 e os 26 meses de idade. Resultados semelhantes foram recentemente reportados por Rowe (2008) e Hurtado, Marchman e Fernald (2008).

Rowe (2008) investigou a complexidade da fala materna medida em termos do seu MLU, bem como da quantidade de palavras (número total de palavras - tokens - e número de palavras diferentes - types) presentes nela. Participaram do estudo quarenta $\mathrm{e}$ cinco crianças de 2,6 anos de idade e suas mães. Os dados foram coletados enquanto as mães interagiam com suas crianças em contextos de vida diária (e.g., durante refeições e lanches, enquanto as mães liam para seus filhos ou brincavam com eles). Para cada díade, 90 minutos de interação foram gravados. O vocabulário receptivo infantil foi avaliado um ano depois, quando as crianças estavam com 3,6 anos, através do Peabody Picture Vocabulary Test-III (DUNN; DUNN, 1997). Os autores encontraram uma correlação significativa entre o MLU, o número total de palavras e sua diversidade na FDC e o vocabulário compreensivo das crianças aos 3,6 anos, mesmo depois de haverem controlado o efeito de diferenças no vocabulário compreensivo das crianças aos 2,6 anos. Hurtado et al. (2008) encontraram resultados semelhantes em um estudo com crianças falantes de espanhol. Esses pesquisadores investigaram a relação entre a linguagem materna e o vocabulário expressivo posterior da criança em uma amostra de vinte e sete crianças. Os resultados mostraram que a diversidade de palavras (types) na linguagem materna, quando as crianças tinham 18 meses de idade, predisse o vocabulário infantil aos 24 meses.

Hoff e Naigles (2002) também encontraram correlações significativas entre medidas da FDC (diversidade de palavras e MLU) e da linguagem infantil. Os autores investigaram uma amostra de sessenta e três crianças em duas ocasiões diferentes. $\mathrm{Na}$ primeira ocasião, as crianças tinham entre 18 e 29 meses de idade. A segunda ocasião ocorreu 10 semanas depois. Nas duas ocasiões foram obtidas medidas da fala infantil; já a fala materna foi obtida apenas na primeira ocasião. A FDC foi analisada quanto ao número de sentenças produzidas, à variação dos tipos de palavras, à frequência total das palavras e ao MLU. A fala infantil foi classificada em função do tamanho do vocabulário e do MLU. Embora a quantidade de enunciados ou palavras produzidas pela mãe não tenha se correlacionado com o vocabulário infantil, tanto o MLU quanto o número de palavras diferentes produzidas pelas mães contribuíram para o desenvolvimento do vocabulário da criança. Entretanto, após controle do efeito de diferenças no vocabulário infantil na primeira ocasião, apenas o MLU materno continuou a contribuir significativamente para o desenvolvimento do vocabulário. 
Em um estudo que se destaca pela sua duração, Roy et al. (2009) mostraram que a frequência com que uma palavra aparece no input infantil está diretamente relacionada à sua aquisição pela criança. Esses pesquisadores acompanharam uma criança dos 9 aos 24 meses de idade e, nesse período, a FDC foi registrada, totalizando 4.260 horas de filmagem. De acordo com eles, a frequência com que as palavras apareciam na fala materna correlacionou-se negativamente com a idade de aquisição dessas palavras pela criança. Ou seja, quanto maior a frequência de uma palavra na FDC, mais cedo ela aparece no vocabulário da criança.

Brent e Siskind (2001) investigaram em que medida o uso de enunciados de uma única palavra (e.g., "come" vem, "go" vai, "now" agora, "up" cima, etc.) auxiliam a aquisição do vocabulário pela criança. Oito mães e seus bebês participaram do estudo. O bebês foram acompanhados durante seis meses, entre 9 e 18 meses de idade, sendo seu vocabulário avaliado aos 12, 15 e 18 meses, através do Inventário MacArthur de Desenvolvimento Comunicativo (FENSON et al., 1993). Para a mãe foi entregue um gravador com a orientação de que ficasse com ele durante um período de aproximadamente 90 minutos por dia. Posteriormente, os enunciados maternos dirigidos às crianças entre os 9 e 15 meses de idade e os enunciados das crianças foram transcritos. Da fala materna, os autores selecionaram todas as palavras que, além de aparecerem dentro de sentenças, foram também enunciadas isoladamente ao menos uma vez. Palavras referentes a rotinas sociais (e.g., "bye-bye" tchau, "Hi" oi etc.) foram excluídas. Os resultados mostraram que 9\% da FDC é constituída por enunciados de uma única palavra, e que $40 \%$ das palavras produzidas pelas crianças aos 12 meses e $27 \%$ das palavras produzidas pelas crianças aos 18 meses correspondia a palavras que haviam sido produzidas isoladamente pela mãe. Regressões logísticas foram realizadas, sendo que as variáveis independentes foram (1) a frequência com que a mãe produzia uma determinada palavra isoladamente e (2) a frequência total com que a mãe produzia essa mesma palavra isoladamente ou não; a variável dependente era a presença da palavra em questão no vocabulário das crianças aos 12 e aos 18 meses. A frequência total com que as crianças ouviam uma determinada palavra não foi um preditor significativo da produção dessa palavra pelas crianças, mas a frequência com que uma determinada palavra era ouvida isoladamente foi um preditor significativo de sua produção posterior pelas crianças.

Finalmente, alguns estudos avaliaram a influência da FDC na aquisição do vocabulário inicial por crianças com desenvolvimento atípico. Cardoso-Martins (1984) investigou crianças portadoras da Síndrome de Down e Girolametto et al. (1999), crianças com atraso de linguagem.

Cardoso-Martins (1984) analisou a relação entre a fala materna dirigida à criança e a aquisição inicial do vocabulário por crianças portadores da síndrome de Down (3 meninos e 3 meninas). A autora encontrou resultados semelhantes aos de Nelson (1973), i.e., as mães com estilos dialógicos tenderam a ter crianças com vocabulário produtivo e receptivo maior do que aquelas que apresentaram um estilo diretivo. No início do estudo de Cardoso-Martins (1984), a idade das crianças variava entre 16 e 19 meses de idade (idade mental entre 8 e 14 meses). As visitas aconteceram a cada 6 semanas, por um período que variou entre 14 e 21 meses. Em cada visita, a mãe e seu(sua) filho(a) foram filmados(as) brincando por 30 minutos com um conjunto fixo de brinquedos. Os enunciados maternos foram classificados em categorias típicas de um 
estilo diretivo (e.g., comandos para ação, questões fechadas e referências negativas ao comportamento da criança) e dialógico (questões abertas e referências positivas ao comportamento da criança). O vocabulário produtivo e receptivo da criança também foi avaliado em cada visita. Análises de correlação entre a fala materna e o vocabulário da criança foram calculadas para a visita que ocorreu quando as crianças tinham em torno de 27 meses de idade, pois até os 19 meses as crianças ainda não produziam nenhuma palavra. De uma maneira geral, os enunciados característicos do estilo diretivo correlacionaram-se negativamente com o vocabulário produtivo e receptivo da criança. O contrário foi observado para os enunciados característicos de um estilo dialógico, os quais tenderam a se correlacionar positivamente com ambas as medidas do vocabulário infantil.

Como observado anteriormente, Girolametto et al. (1999) estavam interessados em investigar o impacto da FDC no desenvolvimento da linguagem de crianças com atraso na aquisição do vocabulário. Doze crianças participaram do estudo, todas com atraso no desenvolvimento do vocabulário expressivo; não houve um grupo controle. Amostras da fala materna e infantil foram coletadas em um contexto de brincadeira livre em duas ocasiões: quando as crianças tinham entre 25 e 30 meses de idade, e quatro meses depois. A fala materna foi codificada quanto ao número total de enunciados, à frequência de palavras enunciadas por minuto, à razão entre o número de palavras diferentes (types) e o número total de palavras (token), ao MLU, ao número de nomeações em episódios de atenção conjunta entre a mãe e a criança, e ao número de imitações, interpretações (e.g., quando a criança balbucia algo e a mãe, utilizando o contexto, infere a que a criança está se referindo), e expansões dos enunciados das crianças feitas pela mãe. A fala infantil foi codificada quanto ao número total de enunciados, número de palavras diferentes (types) e número de enunciados com duas ou mais palavras (e.g. "Red hat" Chapéu vermelho; "Boy has a hat" O menino tem um chapéu). A velocidade da fala materna na primeira ocasião correlacionou-se negativamente com o vocabulário expressivo das crianças na segunda ocasião. Por outro lado, os enunciados maternos contingentes à fala da criança (i.e., as imitações, interpretações, nomeações e expansões) na primeira ocasião correlacionaram-se positivamente com todas as medidas da fala infantil na segunda ocasião, mesmo após o controle do vocabulário expressivo e receptivo das crianças. De acordo com Girolametto et al. (1999), o primeiro resultado poderia ser explicado pelo fato de as mães com uma alta frequência de palavras por minuto provavelmente apresentaram um discurso muito rápido, dificultando identificação das palavras pela criança. Os autores não encontraram correlações significativas entre as medidas da linguagem infantil e a frequência e variedade de palavras na FDC e seu MLU.

\section{DISCUSSÃO E CONSIDERAÇÕES FINAIS}

Considerando todos os estudos aqui descritos, parece inquestionável que algumas características da fala materna auxiliam os bebês a segmentar a fala em palavras. Este é o caso, por exemplo, da prosódia exagerada (e.g., THIESSEN et al., 2005; SINGH et al., 2009) e da existência de palavras muito frequentes na FDC (BORTFELD et al., 2005). Além disso, conforme discutiremos a seguir, alguns aspectos da FDC, ou do 
ambiente linguístico de uma maneira geral, também parecem contribuir para a aquisição do vocabulário pelas crianças.

Em linhas gerais, a variabilidade e frequência de palavras na FDC, bem como a presença de enunciados mais longos (e.g., HURTADO et al., 2008; HUTTENLOCHER et al., 1991; ROWE, 2008), parecem contribuir positivamente para o desenvolvimento inicial do vocabulário infantil. Além disso, o estilo de interação materna dialógico/referencial também mostrou ter um impacto positivo na aquisição da linguagem pelas crianças (CARDOSO-MARTINS, 1984; NELSON, 1973).

Contudo, é preciso cautela na interpretação desses resultados. A imensa maioria desses estudos foi de natureza correlacional, o que não nos permite afirmar que a presença dessas características desempenha um papel causal na aquisição do vocabulário pelas crianças. Com efeito, a existência dessa relação também é consistente com a hipótese de que crianças com um maior vocabulário acabam por estimular suas mães a usar uma linguagem mais rica e variada. Alguns estudos enfrentaram esse problema através de análises longitudinais, em que a linguagem materna em uma determinada ocasião foi correlacionada com o desenvolvimento posterior do vocabulário pela criança. No entanto, mesmo esse design deixa em aberto a possibilidade de que a correlação entre as duas variáveis tenha sido mediada por uma terceira variável qualquer como, por exemplo, o nível de desenvolvimento cognitivo da criança.

Com essas ressalvas em mente, é possível observar que algumas características do input linguístico parecem contribuir para o desenvolvimento do vocabulário infantil. Entre as características que parecem ser benéficas estão o estilo de interação, o qual mostrou influenciar o crescimento do vocabulário infantil de crianças desenvolvendo-se tipicamente (NELSON, 1973), ou não (CARDOSO-MARTINS, 1984), ao longo do segundo ano de vida. Nos estudos de Nelson (1973) e Cardoso-Martins (1984), as crianças cujas mães demonstraram um estilo referencial/dialógico de comunicação (maior número de nomeações e perguntas) apresentaram maiores ganhos na aquisição do vocabulário. Pode-se supor que essas mães eram mais responsivas ao foco da atenção da criança. Há, de fato, evidência de que a nomeação de objetos sob o foco da atenção das crianças (em contraposição a objetos fora do seu foco de atenção) contribui para o desenvolvimento do vocabulário ao longo do segundo ano de vida (TOMASELLO; FARRAR, 1986).

Por outro lado, um número elevado de comandos na FDC sugere um descompasso entre os enunciados maternos e o foco de atenção da criança. Não é surpreendente, portanto, que tanto Nelson (1973) como Cardoso-Martins (1984) tenham encontrado uma relação negativa entre o estilo diretivo de comunicação materna, caracterizado por uma incidência relativamente elevada de comandos, referências negativas ao comportamento da criança e questões fechadas, e o crescimento do vocabulário infantil.

Ao longo do trabalho também foi possível notar que a quantidade e a diversidade de palavras na FDC, ou seja, um ambiente linguístico mais rico e diverso, parece ter um impacto na aquisição do vocabulário infantil (GIROLAMETTO et al., 1999; HOFF; NAIGLES, 2002; HURTADO et al., 2008; HUTTENLOCHER et al., 1991; ROWE, 2008). 
Torna-se importante ressaltar, porém, que a maioria dos estudos longitudinais investigando o impacto da FDC na aquisição inicial do vocabulário não controlou o efeito de diferenças no vocabulário inicial das crianças. Um estudo que realizou tal controle foi o de Hoff e Naigles (2002). Essas autoras mostraram que após esse controle, apenas o MLU continuou contribuindo para a aquisição do vocabulário, sugerindo que a presença de certas construções linguísticas pode desempenhar um papel mais importante na aquisição do vocabulário do que a frequência e/ou variedade de palavras no ambiente linguístico da criança.

O efeito positivo encontrado para o MLU também sugere que certas construções linguísticas podem contribuir mais para a aquisição do vocabulário do que enunciados de uma única palavra. De que maneira isso poderia ser explicado? Suponhamos, por exemplo, uma situação em que a mãe diz "portinha" ao fechar a porta de uma casa de brinquedo. Nesse exemplo, não seria surpreendente se a criança inferisse que a mãe estava se referindo à ação de fechar a porta e não à porta em si. Por outro lado, enunciados do tipo "olha a portinha", "olha a casinha", "olha a bolinha", etc., são menos ambíguos. A razão disso é que a expressão "olha a" é geralmente seguida de um substantivo. Em outras palavras, esses e outros tipos de construção linguística podem fornecer pistas importantes sobre a categoria gramatical das palavras e, dessa forma, contribuir para a apreensão do significado de palavras desconhecidas. De fato, Gómez e Maye (2005) mostraram que crianças de apenas 15 meses de idade são sensíveis a esses padrões no input linguístico. Faz sentido, portanto, que Cardoso-Martins (1984) tenha encontrado uma correlação positiva entre o uso de dêixis (frases do tipo "Esse é o cavalinho"; "Essa aqui é a bolinha") na fala materna e o desenvolvimento do vocabulário no segundo ano de vida.

A importância de certas construções linguísticas não significa que enunciados de palavras isoladas não sejam importantes para a aquisição do vocabulário. Com efeito, há evidência de que a frequência com que uma determinada palavra aparece na FDC contribui para a sua aquisição pela criança, pelo menos quando ela também aparece em outros tipos de construção linguística (BRENT; SISKIND, 2001; ROY et al., 2009)

A maioria dos estudos sobre a relação entre a FDC e o desenvolvimento do vocabulário limitou-se a avaliar o impacto de características gerais da fala materna (e.g., o MLU, a presença de dêixis). Ao que tudo indica, apenas os estudos de Brent e Siskind (2001) e Roy et al. (2009) avaliaram a correlação entre a frequência e o uso de uma determinada palavra na linguagem materna e o uso dessa palavra pela criança.

Como os estudos de Brent e Siskind (2001) e Roy et al. (2009) sugerem, análises considerando a relação entre palavras ou construções específicas na fala materna e na fala da criança oferecem um caminho promissor para estudos futuros interessados em abordar o impacto da frequência de itens específicos na FDC no uso desses itens pelas crianças.

É possível perceber algumas lacunas no conhecimento em torno da relação entre a FDC e a aquisição inicial do vocabulário pelas crianças. Por exemplo, a maioria dos estudos foi realizada com mães e crianças falantes do inglês. Há uma clara ausência de estudos investigando a FDC e seu impacto na aquisição inicial da linguagem em outras línguas, incluindo o português. Uma vez que as línguas diferem entre si em relação aos 
tipos de construção linguística utilizados, tais estudos poderiam contribuir para elucidar quais aspectos da linguagem materna realmente contribuem para o desenvolvimento inicial da linguagem. Como mencionado acima, a despeito do interesse que a interação comunicativa entre as mães e seus bebês tem despertado entre os pesquisadores brasileiros, não encontramos nenhum estudo que tenha investigado os estilos de interação mãe-criança e seu impacto na aquisição inicial da linguagem.

Conforme observamos anteriormente, quase todos os estudos existentes na literatura empregaram uma metodologia correlacional. Estudos futuros empregando uma metodologia experimental são, portanto, necessários para nossa compreensão do papel desempenhado por diferentes características da FDC no desenvolvimento da linguagem pela criança.

\section{REFERÊNCIAS}

ALBIN, D.D.; ECHOLS, C. H. Stressed and word-final syllables in infant-directed speech. Infant behavior en development, v. 19, n. 4, p. 401-418, oct./dec. 1996.

AQUINO, L. de S. B.; SALOMÃO, N. M. R. Estilos diretivos maternos apresentados a meninos e meninas. Estudos de Psicologia, Natal, v. 10, n. 2, p. 223-230, mai./aug. 2005.

ASLIN, R. N.; SAFFRAN, J. R.; NEWPORT, E. L. Computation of conditional probability statistics by 8 month-old infants. Psychological Science, v. 9, n. 4, p. 321-324, jul. 1998.

BARROS, A. L. M. de C.; CAVALCANTE, M. C. B. Manhês: qualidade vocal e deslocamentos na dialogia mãe-bebês. In: FOLTRAN, M. J.; NETO, J. B.; NEGRI, L.; WACHOWICZ, T. C.; SILVA, A. H. P.; GUIMARÃES, M. (Orgs.). Anais do VII Congresso Internacional da Abralin. Curitiba, PR: Associação Brasileira de Linguística. 2011. p. 428-439.

BRAZ, F. de S.; SALOMÃO, N. M. R. A fala dirigia a meninos e meninas: um estudo sobre o input materno e suas variações. Psicologia Reflexão e Crítica, v. 15, n. 2, p. 333-344. 2002.

BORTFELD, H.; MORGAN, J.; GOLINKOFF, R. M.; RATHBUN, K. Mommy and me: Familiar names help lanch babies into speech-stream segmentation. Psychological Science, v. 16, n. 4, p. 298-304, apr. 2005.

BREDVAD-JENSEN, A. C. On prosodic variation in child directed speech in Swedish. TMH-QPSR, v. 37, n. 2, p. 67-68, may. 1996.

BRENT, M. R.; SISKIND, J. M. The role of exposure to isolate words in early vocabulary development. Cognition, v. 81, n. 2, p. 33-44, sep. 2001.

BROOKS, p. J.; KEMPE, V. Language development. United Kingdom: BPS Blackwell, 2012. 400 p.

BRUM-DE-PAULA, M. R. Broto da fala: O papel da prosódia no desenvolvimento da linguagem. Re Vel, v. 8, n. 15, p. 82-94, ago. 2010.

CAMERON-FAULKNER, T.; LIEVEN, E.; TOMASELLO, M. A construction based analysis of child directed speech. Cognitive Science, v. 27, n. 6, p. 843-873, nov./dec. 2003.

CARDOSO-MARTINS, C. Early vocabulary acquisition by down syndrome children: The roles of cognitive development and maternal language input. 1984. 552 f. PhD Dissertation. University of Illinois, Urbana-Champaign, United States, 1984.

CHOMSKY, N. A. Aspects of the theory of syntax. Cambridge, Massachusetts: MIT Press, 1965. $251 \mathrm{p}$. The minimalist program. Cambridge, Massachusetts: MIT Press, 1995. 420 p. Novos horizontes no estudo da linguagem. DELTA, v. 13, n. especial, p. 51-74. 1997.

COOPER, R. P.; ASLIN, R. N. Preference for infant-directed speech in the first month after birth. Child Development, v. 61, n. 5, p. 1584-1595, oct. 1990.

DADALTO, E. V.; GOLDFELD, M. Características do maternalês em duas crianças de idades distintas. Distúrbios da Comunicação, v. 18, n. 2, p. 201-208, ago. 2006. 
DUNN, L. M.; DUNN, L. M. Peabody picture vocabulary test. Third Edition. Circle Pines, MN: American Guidance Service, 1997.

FERGUSON, C. A. Baby talk as a simplified register. In SNOW, C. E.; FERGUSON, C. A. (Eds). Talking to children: Language input and acquisition. Cambridge, United Kingdom: Cambridge University Press, 1977. p. 31-49.

FERNALD, A. Four-month infants prefer listen to motherese. Infant Behavior and Development, v. 8, n. 2, p. 181-195, apr./jun. 1985.

. Intonation and communicative intent in mothers' speech to infants: is the melody the message? Child Development, v. 60, n. 6, p. 1497-1510, dec. 1989.

FERNALD, A.; MAZZIE, C. Prosody and focus in speech to infants and adults. Developmental Psychology, v. 27, n. 2, p. 209-221, mar. 1991.

FERNALD, A.; MCROBERTS, G. W.; SWINGLEY, D. Infants' developing competence in recognizing and understanding words in fluent speech. In: WEISSENBORN, J; HOHLE, B. (Eds.). Approaches to bootstrapping: Phonological, lexical, syntactic and neurophysiological aspects of early language acquisition. Amsterdam, Netherlands: John Benjamins Publishing Company, 2001. p. 97-123.

FERNALD, A.; SIMON, L. Expanded intonation contours in mothers' speech to newborns. Developmental Psychology, v. 20, n. 1, p. 104-113, jun. 1984.

FERREIRA, S. S. Por que falar ao bebê se ele não compreende? In: CAMAROTTI, M. C. (Org.). Atendimento ao bebê: Uma abordagem interdisciplinar. São Paulo: Casa do Psicólogo, 2001. p. 97-116. FONSÊCA, p. N. da; SALOMÃO, N. M. R. Análise comparativa das falas materna e paterna dirigida às crianças. Paidéia, v. 15, n. 30, p. 70-91, jan./apr. 2005.

GIROLAMETTO, L.; WEITZMAN, E.; WIGS, M.; PEARCE, p. S. The relationship between maternal language measures and language development in toddlers with expressive vocabulary delays. American Journal of Speech Language Pathology, v. 8, n. 4 p. 364-374, nov. 1999.

GLEITMAN, L. R.; NEWPORT, E. L.; GLEITMAN, H. The current status of motherese hypothesis. Journal of Child Language, v. 11, n. 1, p. 43-79, feb. 1984.

GÓMEZ, R. L.; MAYE, J. The developmental trajectory of nonadjacent dependency learning. Infancy, v. 7, n. 2, p. 183-206, mar. 2005.

GOUT, A.; CHRISTOPHE, A. O papel do bootstrapping prosódico na aquisição de sintaxe do léxico. In: CORRÊA, L. M. S. (Org.). Aquisição da linguagem e problemas do desenvolvimento linguístico. Rio de Janeiro: Edições Loyola, 2006. p. 103-127.

HOFF, E. (2005). Language development. 3rd ed. Florida, United States: Wadsworth, 2005. 480 p.

HOFF, E.; NAIGLES, L. How children use input to acquire a lexicon. Child Development, v. 73, n. 2, p. 418-433, mar./apr. 2002.

HOFF-GINSBERG, E.; SHATZ, M. Linguistic input and the child's acquisition of language.

Psychological Bulletin, v. 92, n. 1, p. 3-26, jul. 1982.

HURTADO, N.; MARCHMAN, V. A.; FERNALD, A. Does input influence uptake? Links between maternal talk, processing speed and vocabulary size in Spanish learning children. Developmental Science, v. 11, n. 6 , p. 803-895, nov. 2008.

HUTTENLOCHER, J.; HAIGHT, W.; BRYK, A.; SELTZER, M.; LYONS, T. Early vocabulary growth: Relation to language input and gender. Developmental Psychology, v. 27, n. 2, p. 236-248, mar. 1991.

JUSCZYK, p. How infants begin to extract words from speech. Trends in Cognitive Science, v. 3, n. 9, p. 323-328, sep. 1999.

JUSCZYK, p. ; ASLIN, p. N. Infants detection of the sound patterns of words in fluent speech. Cognitive Psychology, v. 29, n. 1, p. 1-23, aug. 1995.

KANPEM, A. van; PARMAKSIZ, G.; VJVER, R. van de; HOHLE, BMetrical and statistical cues for word segmentation: vowel harmony and word stress as cues to word boundaries by 6-and-9-month-old Turkish learners. In GARRAVÓ, A.; FREITAS, M J. Freitas (Eds.), Language acquisition and development. Cambridge, United Kingdom: Cambridge Scholar Publishing, 2008. p. 313-324.

KEMLER-NELSON, D.; JUSCZYK, p. W.; Mandel, D. R.; Myers, J.; Turk, A. E.; Gerken, L. The headturn preference procedure for testing auditory perception. Infant Behavior e Development, v. 18, n. 1, p. 111-116, jan./mar.1995.

BARBOSA, Poliana Gonçalves; CARDOSO-MARTINS, Cláudia. Uma revisão dos estudos sobre a fala dirigida à criança e suas implicações para a aquisição inicial do vocabulário. Linguagem em (Dis)curso - LemD, Tubarão, SC, v. 14, n. 1, p. 195-210, jan./abr. 2014. 
MAHDHOUI, A.; CHETOUANI, M.; ZONG, C. Motherese detection based on segmental and suprasegmental features. In Conference on Pattern Recognition, 19, 2008. Summaries... Ivry sur Seine, France: IEEE, 2008. $4005 \mathrm{p}$.

NAME, M. C.; CORRÊA, L. M. S. Explorando a escuta, o olhar e o processamento sintático: metodologia experimental para o estudo da aquisição da língua materna em fase inicial. In: CORRÊA, L. M. S. (Org.). Aquisição da linguagem e problemas do desenvolvimento linguístico. Rio de Janeiro: Edições Loyola, 2006. p. 79-100.

NELSON, K. Structure and strategy in learning to talk. Monographs of the Society for Research in Child Development, v. 38, n. 1-2, s. 149. Chicago, United States: University of Chicago Press, 1973.

NEWPORT, E. L. Motherese: The speech of mothers to young children. 1995. $196 \mathrm{f}$. PhD Dissertation, Center for Human Information Processing, University of California, San Diego, 1995.

NEWPORT, E. L.; GLEITMAN, H.; GLEITMAN, L. R. Mother, I'd rather do it myself: Some effects and non-effects of maternal speech style. In: SNOW, C. E.; FERGUSON, C. A. (Eds.). Talking to children: Language input and acquisition. Cambridge, United Kingdom: Cambridge University Press, 1977. p. 109-149.

PÊSSOA, L. F.; SEIDL-DE-MOURA, M. L. Características pragmáticas da fala materna em díades mãebebê (aos cinco e vinte meses). Arquivos Brasileiros de Psicologia, v. 60, n. 1, p. 82-95, abr. 2008.

Fala materna dirigida à criança em cenários comunicativos específicos: um estudo longitudinal. Psicologia: Teoria e Pesquisa, v. 27, n. 4, p. 439-447, out./dez. 2011.

PÊSSOA, L. F.; SEIDL-DE-MOURA, M. L.; OLIVA, A. D. A análise da fala materna dirigida a bebês em duas etapas do desenvolvimento. Psicologia em Pesquisa, v. 2, n. 2, p. 74-86, jul./dez. 2008.

ROWE, M. L. Child-directed speech: Relation to socioeconomic status, knowledge of child development and child vocabulary skill. Journal of Child Language, v. 35, n. 1, p. 185-205, feb. 2008.

ROY, B. C., FRANK, M. C.; ROY, D. Exploring word learning in a high-density longitudinal corpus. In: Annual Cognitive Science Conference, 31, 2009. Summaries... Amsterdam, Netherlands: Cognitive Science Society. 3280 p.

SAFFRAN, J.; ASLIN, R.; NEWPORT, E. Statistical learning by 8-month-old infants. Science, v. 274, n. 5294, p. 1926-1928, dec. 1996.

SINGH, L.; NESTOR, S.; PARIKH, C.; YULL, A. Influences of infant directed speech on early word recognition. Infancy, v. 14, n. 6, p. 654-666, nov./dec. 2009.

SNOW, C. E. Mothers speech to children learning language. Child Development, v. 43, n. 2, p. 549-565, jun. 1972.

Mothers' speech research: From input to interaction. In: SNOW, C. E.; FERGUSON, C. A. (Eds.). Talking to children: Language input and acquisition. Cambridge, United Kingdom: Cambridge University Press, 1977. p. 31-49.

SODERSTROM, M. Beyond baby talk: re-evaluating the nature and content of speech input to preverbal infants. Developmental Review, v. 27, n. 4, p. 501-532, dec. 2007.

THIESSEN, E. D.; HILL, E. A.; SAFFRAN, J. R. Infant-directed speech facilitates word segmentation. Infancy, v. 7, n. 1, p. 53-71, jan. 2005.

TOMASELLO, M. Construction grammar for kids. Construction, spv. 1, p. 1-23. 2006.

. Language development. In GOSWAMI, U. (Org.), The WILEY-BLACKWELL handbook of childhood cognitive development. Oxford, United Kingdom: Blackwell Publishers, 2011. p. 239-257.

TOMASELLO, M.; FARRAR, M. J. Joint attention and early language. Child Development, v. 57, n. 6, p. 1454-1463, dec. 1986.

VERÁS, R. M.; SALOMÃO, N. M. R. Interações entre díades mãe-criança que apresentam a linguagem expressiva típica e díades de mãe-criança que apresenta a linguagem expressiva atrasada. Interação em Psicologia, v. 9, n. 1, p. 165-176, jan./jul. 2005.

WAXMAN, S. R.; LEDDON, E. M. Early word-learning and conceptual development. In: GOSWAMI, U. (Org.). The WILEY-BLACKWELL handbook of childhood cognitive development. Oxford, United Kingdom: Blackwell Publishers, 2011. p. 180-208.

Recebido em: 30/03/13. Aprovado em: 10/12/13.

BARBOSA, Poliana Gonçalves; CARDOSO-MARTINS, Cláudia. Uma revisão dos estudos sobre a fala dirigida à criança e suas implicações para a aquisição inicial do vocabulário. Linguagem em (Dis)curso - LemD, Tubarão, SC, v. 14, n. 1, p. 195-210, jan./abr. 2014. 
Title: A review of studies on child-directed speech and its implications for early vocabulary acquisition

Author: Poliana Gonçalves Barbosa; Cláudia Cardoso-Martins

Abstract: Motivated by Chomsky's reasoning about the poverty of the linguistic input, a number of researchers have investigated the characteristics of child-directed speech (CDS) and their impact on children's language acquisition. In this article, we review studies that investigated the relationship between CDS and children's initial vocabulary acquisition. Results suggest that CDS's style, mean length utterance, frequency and variety of words have an impact on early vocabulary acquisition. These results have however to be interpreted with caution. In general, only correlational studies have investigated the contribution of CDS to early vocabulary development. In addition, most of them were conducted with English-speaking children. Experimental studies as well as studies including children speaking other languages are clearly necessary to further investigate this issue.

Keywords: Language acquisition. Child-directed speech. Vocabulary

Título: Una revisión de estudios sobre el habla dirigida a niños y sus implicaciones para adquisición inicial del vocabulario

Autores: Poliana Gonçalves Barbosa; Cláudia Cardoso-Martins

Resumen: Investigadores motivados por el argumento de Chomsky sobre la pobreza del input lingüistico han investigado las características del habla dirigida a niños (HDN) y su impacto en el desarrollo del lenguaje infantil. En esa retrospectiva, revisamos estudios que investigaron la relación entre el HDN y el desarrollo inicial del vocabulario por los niños. Los resultados sugieren que variaciones de estilo, en la longitud media de las enunciaciones y en la frecuencia y variedad de palabras en el HDN tiene impacto en la adquisición inicial del vocabulario infantil. Sin embargo, eses resultados deben ser interpretados con cautela. De manera general, sólo estudios correlacionados investigaron la contribución del HDN para el desarrollo del vocabulario. Además, la mayoría de ellos fue conducida con niños hablantes de inglés. Estudios experimentales e incluyendo niños hablantes de otras lenguas son claramente necesarios para investigar esa cuestión.

Palabras-clave: Adquisición de lenguaje. Habla dirigida a niños. Vocabulario.

BARBOSA, Poliana Gonçalves; CARDOSO-MARTINS, Cláudia. Uma revisão dos estudos sobre a fala dirigida à criança e suas implicações para a aquisição inicial do vocabulário. Linguagem em (Dis)curso - LemD, Tubarão, SC, v. 14, n. 1, p. 195-210, jan./abr. 2014. 